\title{
ON THE FLOQUET PROBLEM FOR QUASIPERIODIC SYSTEMS
}

\author{
J. A. MURDOCK
}

\begin{abstract}
The problem of reducing $\dot{x}=A(t) x$, where $A(t)$ is a quasiperiodic $n \times n$ matrix, to a system with constant coefficients is studied by means of an associated linear partial differential equation. A necessary and sufficient condition is given which, while not always computable, appears to provide a new approach to this classical problem.
\end{abstract}

1. Introduction. An $n \times n$ complex-valued matrix function $A(t)$ is termed quasiperiodic with $k$ incommensurable frequencies $\omega_{1}, \ldots, \omega_{k}$ if $A(t)=$ $\hat{A}\left(\omega_{1} t, \ldots, \omega_{k} t\right)$ where $\hat{A}\left(\theta_{1}, \ldots, \theta_{k}\right)$ is continuous and periodic with period $2 \pi$ in each argument. A linear system of differential equations $\dot{x}=A(t) x$, where $A(t)$ is quasiperiodic, is called reducible if there is a differentiable quasiperiodic matrix $P(t)$ (with the same frequencies) such that the change of variables $x=P(t) y$ results in $\dot{y}=B y$ where $B$ is a constant matrix. If $k=1$ then all systems are reducible by Floquet theory; if $A(t)$ and $P(t)$ are allowed to be almost (as opposed to quasi) periodic then not all systems are reducible [1].

In order to simplify the notation the results will be stated and proved for the case $k=2$; the immediate generalization to arbitrary $k$ will be briefly indicated in $\S 4$. Thus, let $A(t)=\hat{A}(\omega t, \nu t)$ with $\omega / \nu$ irrational and consider, along with

$$
\dot{x}=A(t) x,
$$

the partial differential equation

$$
\omega(\partial \hat{u} / \partial \theta)+\nu(\partial \hat{u} / \partial \varphi)=\hat{A}(\theta, \varphi) \hat{u} .
$$

By analogy with Floquet theory we shall introduce a nonsingular fundamental matrix solution $\hat{X}(\theta, \varphi)$ of (2) and, associated with such a solution, we define two monodromy matrices $\hat{C}, \hat{D}$ by

$$
\begin{aligned}
& \hat{C}(\theta, \varphi)=\hat{X}^{-1}(\theta, \varphi) \hat{X}(\theta+2 \pi, \varphi), \\
& \hat{D}(\theta, \varphi)=\hat{X}^{-1}(\theta, \varphi) \hat{X}(\theta, \varphi+2 \pi) .
\end{aligned}
$$

In general these matrices depend upon $\theta$ and $\varphi$. Our principal result is that system (1) is reducible if and only if (2) possesses a fundamental solution whose monodromy matrices are constant. This result is proved in $\$ 2$. In $\$ 3$ we

Received by the editors January 5, 1976.

AMS (MOS) subject classifications (1970). Primary 34A30.

Key words and phrases. Quasiperiodic, linear ordinary differential equations. 
make a tentative effort to exploit this condition by relating it to asymptotic properties of the solution of (1).

Our methods are very close to those of classical Floquet theory and unlike any attempts on this problem of which the author is aware. A general reference for the subject is [1].

2. The main theorem. The characteristic base curves of (2) are the straight lines of slope $\nu / \omega$ in the $(\theta, \varphi)$ plane. The change of variables $\theta=\nu r+\omega s$, $\varphi=-\omega r+\nu s$ reduces (2) to the system of ordinary differential equations

$$
\partial u / \partial s=A(r, s) u
$$

where we have adopted the convention that $f(r, s)=\hat{f}(\theta, \varphi)$ for any function defined in the $(\theta, \varphi)$ plane. System (4) contains $r$ as a parameter, reduces to (1) for $r=0$, and constitutes a concrete realization of the hull of (1), in the language of almost-periodic theory.

LEMMA 1. Let $\hat{P}(\theta, \varphi)$ be a differentiable periodic matrix function of $\boldsymbol{\theta}$ and $\varphi$, and let $B$ be a constant matrix. The following are equivalent:

(i) The change of variables $x=\hat{P}(\omega t, v t) y$ reduces (1) to $\dot{y}=B y$.

(ii) The change of variables $\hat{u}=\hat{P}(\theta, \varphi) \hat{v}$ reduces (2) to $\omega \partial \hat{v} / \partial \theta+\nu \partial \hat{v} / \partial \varphi$ $=B \hat{v}$.

(iii) The change of variables $u=P(r, s) v$ reduces (4) to $\partial v / \partial s=B v$.

Proof. The equivalence of (ii) and (iii) is immediate. Let the result of applying $u=P(r, s) v$ to (4) be written $\partial v / \partial s=B(r, s) v$; then setting $r=0$ gives the same ordinary differential equation as that obtained by applying $x=P(\omega t, v t) y$ to (1). Thus it is clear that statement (iii), which asserts that $B(r, s)$ is constant, implies statement (i). Conversely if (i) holds then $B(r, s)$ is constant on the line $r=0$; since $B(r, s)=\hat{B}(\theta, \varphi)$ is $2 \pi$-periodic in $\theta$ and $\varphi$ it is constant along all lines obtained by shifting the line $r=0$ by $2 \pi$ to the right, left, up, or down, Since this line has irrational slope, $\hat{B}(\theta, \varphi)$ is constant on a dense set and hence everywhere. Thus (i) implies (ii). Q.E.D.

System (4), regarded as an ordinary differential equation with parameter $r$, possesses fundamental matrix solutions $X(r, s)$ : we may let $X(r, 0)$ be any differentiable nonsingular matrix function of $r$, and $X(r, s)$ will be unique, nonsingular, and differentiable everywhere.

Increasing $\theta$ by $2 \pi$ shifts $r$ to $r+\alpha$ and $s$ to $s+\beta$, where $\alpha=2 \pi \nu / \Delta$, $\beta=2 \pi \omega / \Delta, \Delta=\omega^{2}+\nu^{2}$. Similarly, increasing $\varphi$ by $2 \pi$ shifts $r$ to $r-\beta$ and $s$ to $s+\alpha$. Hence the monodromy matrices (3) take the form

$$
\begin{aligned}
& C(r, s)=X^{-1}(r, s) X(r+\alpha, s+\beta), \\
& D(r, s)=X^{-1}(r, s) X(r-\beta, s+\alpha) .
\end{aligned}
$$

LEMma 2. $C(r, s)$ and $D(r, s)$ are independent of $s$; that is, $C(r, s)=C(r)$, $D(r, s)=D(r)$.

Proof. 


$$
\begin{aligned}
(\partial / \partial s) X(r+\alpha, s+\beta) & =A(r+\alpha, s+\beta) X(r+\alpha, s+\beta) \\
& =A(r, s) X(r+\alpha, s+\beta)
\end{aligned}
$$

since $A(\theta, \varphi)$ is $2 \pi$-periodic in $\theta$. Hence, $X(r+\alpha, s+\beta)$ is a fundamental solution of (4), and differs from $X(r, s)$ by a right matrix factor $C(r)$ depending only upon $r$. The proof for $D$ is similar. An alternate proof may be given by differentiating (5) with respect to $s$, using (4) and the adjoint equation satisfied by $X^{-1}$ to obtain $\partial C(r, s) / \partial s=0$. Q.E.D.

The next lemma establishes the commutation properties of the monodromy matrices.

LEMMA 3. $C(r) D(r+\alpha)=D(r) C(r-\beta)$.

Proof.

$$
\begin{aligned}
X(r+\alpha-\beta, s+\beta+\alpha) & =X(r+\alpha, s+\beta) D(r+\alpha) \\
& =X(r, s) C(r) D(r+\alpha)
\end{aligned}
$$

on the other hand

$$
\begin{aligned}
X(r+\alpha-\beta, s+\beta+\alpha) & =X(r-\beta, s+\alpha) C(r-\beta) \\
& =X(r, s) D(r) C(r-\beta) \text { Q.E.D. }
\end{aligned}
$$

We are now ready to prove

THEOREM 1. The following statements are equivalent.

(i) There exists a differentiable periodic matrix function $\hat{P}(\theta, \varphi)$ such that the change of variables $x=\hat{P}(\omega t, v t) y$ reduces (1) to $\dot{y}=B y$, where $B$ is $a$ constant matrix.

(ii) There exists a differentiable fundamental solution $X(r, s)$ of (4) having constant monodromy matrices $C(r)=C, D(r)=D$.

In the case that (i) and (ii) hold, there in fact exists a fundamental solution for which $C=Q^{\beta}, D=Q^{\alpha}$ for a constant matrix $Q$.

Proof. Assume (i). By Lemma 1 the transformation $u=P(r, s) v$ reduces (4) to $\partial v / \partial s=B v$, which possesses a fundamental solution $Y(r, s)=e^{B s}$. Hence (4) has a fundamental solution $X(r, s)=P(r, s) e^{B s}$ whose monodromy matrices we calculate:

$$
\begin{aligned}
C(r) & =X^{-1}(r, s) X(r+\alpha, s+\beta) \\
& =e^{-B s} P^{-1}(r, s) P(r+\alpha, s+\beta) e^{B(s+\beta)}=e^{B \beta}=Q^{\beta}
\end{aligned}
$$

where $Q=e^{B}$; similarly $D(r)=Q^{\alpha}$. Hence the monodromy matrices of this fundamental solution are constant and have the desired form.

Assume (ii). Since the monodromy matrices are constant, the commutation relations (Lemma 3) reduce to $C D=D C$. Choose matrices $R, S$ such that $C=e^{\omega R}, D=e^{\nu S}$, and $R S=S R$. (This may be done, for instance, by functional calculus: slit the complex plane from 0 to $\infty$ along a path which avoids eigenvalues of $C$ and $D$, choose a branch of $\log$ on the slit plane and a 
contour surrounding the eigenvalues of $C$ and $D$, and define

$$
\log C=(2 \pi i)^{-1} \oint(C-z I)^{-1} \log z d z
$$

and $\log D$ similarly; these $\operatorname{logs}$ commute because the resolvents of $C$ and $D$ commute. Note that $R$ and $S$ may be complex even if $C$ and $D$ are real: as in standard Floquet theory it is necessary to double the periods to obtain real results.)

Define $\hat{P}(\theta, \varphi)=\hat{X}(\theta, \varphi) e^{-(\omega \theta R+\nu \varphi S) / 2 \pi}$ and observe that $\hat{P}(\theta, \varphi)$ is $2 \pi-$ periodic in $\theta$ and $\varphi$. We claim that $\hat{P}(\theta, \varphi)$ carries out the desired reduction. From $\theta=\nu r+\omega s, \varphi=-\omega r+\nu s$ and the definition of $\hat{P}(\theta, \varphi)$ it follows that

$$
\begin{aligned}
X(r, s) & =P(r, s) \exp \{\omega(\nu r+\omega s) R+\nu(-\omega r+\nu s) S\} / 2 \pi \\
& =P(r, s) e^{B s} F(r)
\end{aligned}
$$

where $B=\left(\omega^{2} R+\nu^{2} S\right) / 2 \pi$ and $F(r)=\exp \omega \nu r(R-S) / 2 \pi$. Hence if the change of variables $u=P(r, s) v$ is applied to (4) the resulting equation for $v$ will have $e^{B s} F(r)$ as a fundamental solution, from which it follows that $\partial v / \partial s=B v$. Statement (i) follows by Lemma 1. Q.E.D.

As a final remark we observe that the differentiability requirement in Theorem 1 is stronger than necessary. It is meaningful to consider solutions of (4) which are differentiable in $s$ but merely continuous in $r$, and the same may be done for (2) if $\omega \partial / \partial \theta+\nu \partial / \partial \varphi$ is regarded as a directional derivative operator rather than a combination of partial derivatives. In the same way a periodic matrix function $\hat{P}(\theta, \varphi)$ which is continuous and possesses the directional derivative $\omega \partial / \partial \theta+\nu \partial / \partial \varphi$ everywhere, without necessarily possessing partial derivatives $\partial / \partial \theta$ and $\partial / \partial \varphi$, defines a legitimate change of variables. Theorem 1 remains true if "differentiable" is interpreted in this way in statements (i) and (ii).

3. Change of fundamental solution. If system (4) has a fundamental solution $X(r, s)$ for which the monodromy matrices are not constant, is it possible to find another fundamental solution for which they are? Any other fundamental solution has the form $Y(r, s)=X(r, s) K(r)$ where $K$ is a continuous nonsingular matrix function of $r$ (here we allow fundamental solutions which are differentiable only with respect to $s$, as in the last paragraph of $\S 2$ ).

The monodromy matrices of $Y(r, s)$ are obtained from those of $X(r, s)$ by "shifted similarities"

$$
\begin{aligned}
& E(r)=K^{-1}(r) C(r) K(r+\alpha), \\
& F(r)=K^{-1}(r) D(r) K(r-\beta) .
\end{aligned}
$$

We ask: is it possible to choose $K$ so that $E$ and $F$ are constant? Theorem 1 shows that if this is possible then it is also possible to obtain $E=Q^{\beta}$, $F=Q^{\alpha}$, and we shall seek this form, although our results are incomplete and tentative. Without loss of generality we assume $X(r, 0)=I$. 
LEMMA 4. The equations

$$
\begin{aligned}
& K^{-1}(r) C(r) K(r+\alpha)=Q^{\beta}, \\
& K^{-1}(r) D(r) K(r-\beta)=Q^{\alpha},
\end{aligned}
$$

together with $K(0)=I$ define $K$ uniquely on the module of real numbers of the form $m \alpha+n \beta, m$ and $n$ integers. For numbers of this form,

$$
K(m \alpha+n \beta)=X^{-1}(m \alpha+n \beta, m \beta-n \alpha) Q^{m \beta-n \alpha} .
$$

Proof. Clearly $K(0)=I$ together with the equations above give many formally distinct determinations of $K(m \alpha+n \beta)$ for integers $m$ and $n$. Hence existence and uniqueness both follow from direct verification that the expression above satisfies these equations:

$$
\begin{aligned}
& K^{-1}(m \alpha+n \beta) C(m \alpha+n \beta) K((m+1) \alpha+n \beta) \\
&= Q^{n \alpha-m \beta} X(m \alpha+n \beta, m \beta-n \alpha) \\
& \cdot X^{-1}(m \alpha+n \beta, m \beta-n \alpha) X((m+1) \alpha+n \beta,(m+1) \beta-n \alpha) \\
& \cdot X^{-1}((m+1) \alpha+n \beta,(m+1) \beta-n \alpha) Q^{(m+1) \beta-n \alpha}=Q^{\beta},
\end{aligned}
$$

where we have used (5), setting $s=m \beta-n \alpha$ by virtue of Lemma 2 . The second equation may be checked similarly. Q.E.D.

If it is possible to choose $Q$ in such a way that $K$ as constructed above is uniformly continuous on the dense set of numbers of the form $m \alpha+n \beta$, then it follows from Theorem 1 that the system is reducible. It appears that the existence of such a $Q$ is related to asymptotic properties of $X(r, s)$ as $s \rightarrow \infty$ along special sequences $s_{k}$, although the exact relationship is not yet clear. Namely we can show that if $m_{k}, n_{k}$ are selected so that $r_{k}=m_{k} \alpha+n_{k} \beta \rightarrow 0$ as $k \rightarrow \infty$, then $s_{k}=m_{k} \beta-n_{k} \alpha$ is a sequence of $\varepsilon_{k}$-almost periods of (1) with $\varepsilon_{k} \rightarrow 0$. Therefore the hypothesis that $K$ is continuous at zero (and $K(0)=I$ ) implies

$$
\lim _{k \rightarrow \infty} X^{-1}\left(r_{k}, s_{k}\right) Q^{s_{k}} \rightarrow I
$$

and hence $X\left(0, s_{k}\right)$ is nearly $Q^{s_{k}}$ for large $k$. If it could be shown that fundamental solutions of (1) either do or do not have such "asymptotic properties along almost-period sequences", the Floquet problem might be resolved.

4. More frequencies. If $A(t)=\hat{A}\left(\omega_{1} t, \ldots, \omega_{k} t\right)$ in (1) then (2) is replaced by $\left(\omega_{1} \partial / \partial \theta_{1}+\cdots+\omega_{k} \partial / \partial \theta_{k}\right) \hat{u}=\hat{A}\left(\theta_{1}, \ldots, \theta_{k}\right) \hat{u}$. Let $T$ be a $k \times k$ orthogonal matrix whose last column is $\operatorname{col}\left(\omega_{1}, \ldots, \omega_{k}\right)$, and define new coordinates $\left(r_{1}, \ldots, r_{k-1}, s\right)$ by $\operatorname{col}\left(\theta_{1}, \ldots, \theta_{k}\right)=T \operatorname{col}\left(r_{1}, \ldots, r_{k-1}, s\right)$. The system becomes $\partial u / \partial s=A\left(r_{1}, \ldots, r_{k-1}, s\right)$ depending upon $k-1$ parameters $r_{1}, \ldots, r_{k-1}$; this is the analog of (4). There are now $k$ monodromy matrices $C_{i}\left(r_{1}, \ldots, r_{k}\right), i=1, \ldots, k$, which are independent of $s$. All of the 
results of $\$ 2$ carry over in an obvious way, and (1) is reducible if and only if there exists a fundamental solution with constant monodromy matrices.

\section{REFERENCES}

1. A. M. Fink, Almost periodic differential equations, Lecture Notes in Math., vol. 377, Springer-Verlag, Berlin and New York, 1974.

Department of Mathematics, Iowa State Universtty, Ames, Iowa 50011 